Research Article

\title{
Impact of Lockdown on Sleep Quality among Collegiate Students during COVID- 19
}

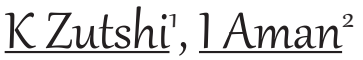 \\ ${ }^{1}$ Associate Professor, Department of Rehabilitation Sciences, Jamia Hamdard University. \\ ${ }^{2}$ Senior Physiotherapist, Fitsol Sports Medicine, New Delhi, India. \\ DOI: https://doi.org/10.24321/2455.9199.202104
}

\section{I $\quad \mathbf{N} \quad \mathbf{F}$}

\author{
Corresponding Author: \\ K Zutshi, Department of Rehabilitation Sciences, \\ Jamia Hamdard University. \\ E-mail Id: \\ zutshi.kalpana@gmail.com \\ Orcid Id: \\ https://orcid.org/0000-0002-3494-5665 \\ How to cite this article: \\ Zutshi K, Aman I. Impact of Lockdown on Sleep \\ Quality among Collegiate Students during \\ COVID-19. Int J HealthCare Edu \& Med Inform. \\ 2021;8(3):4-7. \\ Date of Submission: 2021-04-03 \\ Date of Acceptance: 2021-09-01
}

\section{$\begin{array}{llllllll}A & B & \mathbf{S} & \mathbf{T} & \mathbf{R} & \mathbf{A} & \mathbf{C} & \mathbf{T}\end{array}$}

Background: The Indian population was placed in social isolation due to spread of corona virus. There was a significant increase of psychological distress and symptoms of mental illness in the general population. The aim of this study was to assess the impact of lockdown on sleep quality among collegiate students during COVID-19 pandemic and help healthcare workers to understand the psychological aspect of lockdown and help the community.

Method: It is a cross-sectional survey study. Collegiates were selected for the study. An online questionnaire was sent via email or whatsapp. Data was analyzed and result was obtained.

Result: Result showed that $19.1 \%$ were having very good sleep quality whereas $12.1 \%$ were having bad sleep. Sleep latency was $8.5 \%$ participants slept within $<15$ minutes, $35.5 \%$ participants slept within 16 to 30 minutes, $26.2 \%$ participants took 31 to 60 minutes whereas $29.8 \%$ participants took more than 60 minutes. Sleep efficiency result was: $51.8 \%$ participants were having very good sleep efficiency whereas $7.1 \%$ were having very bad sleep efficiency. $61.7 \%$ participants were having very good sleep duration hours whereas $9.9 \%$ participants were having very bad sleep duration hours. $4 \%$ to $3 \%$ participants did not take sleep medicine in last month whereas $0.7 \%$ had taken medication for sleep for more than thrice in a week. $68.8 \%$ participants had taken sleep medicine once a week and $26.2 \%$ had taken medicine twice a week. $41.1 \%$ participants never had day time dysfunction whereas $5.0 \%$ participants had day time dysfunction.

Conclusion: Lockdown had affected the sleep quality of collegiate students.

Keywords: Sleep, Lockdown, COVID-19, PSQI Scale

\section{Introduction}

There is a greenhorn new public health crises threatening the globe with the emergence and unfold of a try of pair of 2019 novel corona virus (2019-nCoV) or the severe acute metabolism syndrome corona virus 2 (SARS-CoV-2). The virus originated in kooky (bats) and was transmitted to humans in Wuhan, Hubei province, China in December 2019. The malady is transmitted by inhalation or contact with infected droplets and also the fundamental time 
frame ranges from 2 to $14 \mathrm{~d}$. The symptoms are usually fever, cough, raw throat, dyspnea, fatigue, and unease among others. Diagnosis is by confirmation of the virus in respiratory secretions by special molecular tests. Common laboratory findings embrace normal/ low phagocyte counts with elevated serum globulin (CRP). Treatment is actually sustaining. Hindrance entails home isolation of suspected cases and people with gentle sickness and strict infection management measures at hospitals that embrace contact and driblet precautions. ${ }^{1}$

The Indian population was placed in social isolation for pretty much 57 days with the sole permission to depart from home for getting food or medicines. These restrictions greatly modify lifestyles and social relationships creating in many individuals augmented levels of tension also because of the fear of being infected. There was a significant increase of psychopathy and symptoms of mental illness among the overall general population. . $^{2,3}$

Corona viruses are a bunch of viruses belonging to the family of Coronaviridae, enveloped, positive singlestranded massive ribonucleic acid viruses with "humanto-human" transmission through respiratory secretions. The clinical manifestations of COVID-19 comprise fever, cough, nasal congestion, fatigue, and alternative signs of upper tract infections including dyspnoea and severe chest symptoms admire pneumonia and, to date, no specific therapeutic drug has been found. It should be prevented by oftentimes washing hands, carrying a face mask and by social distancing. ${ }^{4}$ The COVID-19 pandemic could be a nonnatural disaster that may have bearing an impact on the mental state and psychosocial conditions of everybody. In keeping with WHO (2020), the emergence of an outbreak caused stress to varied levels of society. Through up to now, there has not been a scientific review of the effects and result of COVID-19 on psychological state, form of studies associated to pandemics (including bird flu and SARS) have shown a negative impact on the mental health of sufferers. ${ }^{5,6}$ This has conjointly restricted the academic activity across the globe. ${ }^{7}$ The national closure has a bearing of quite more than $91 \%$ of the world's student population. Many other countries also bear the local closures which have an impact on voluminous students. ${ }^{8}$

Sleep is interpreted as behavioural and physiological criteria divided into two states: Non-rapid eye movement (NREM) sleep which is subdivided into three classes (N1, N2, N3) and rapid eye movement (REM) which is categorized by rapid eye movement, muscle amyotonia and desynchronized ECG. ${ }^{9}$ Sleep quality is a vital clinical construct, it represents a posh phenomenon that's difficult to stipulate and measure objectively. Sleep quality includes quantitative aspect of sleep, like sleep duration, sleep latency, or number of arousals likewise as more purely subjective aspect such as depth or restfulness of sleep. PSQI is primarily intended to measure sleep quality and to spot good and bad sleepers. ${ }^{10}$ The Pittsburgh Sleep Quality Index (PSQI) is an effective instrument accustomed to measure the quality and patterns of sleep with in the older adult. It discriminates "poor" from "good" sleep by measuring seven areas: subjective sleep quality, sleep latency, sleep duration, habitual sleep efficiency, sleep disturbances, use of sleeping medication, and daytime dysfunction over the last month. The shopper self-rates each of these seven areas of sleep. Scoring of answers is based on a 0 to 3 scale, wherever 3 replicate the negative extreme on the Likert scale. A worldwide sum of 5 or bigger indicates a "poor" sleeper. ${ }^{11}$

The aim of this study was to assess the impact of lockdown on sleep quality among collegiate students during COVID-19 pandemic and help healthcare workers to understand the psychological aspect of lockdown and help the community.

\section{Methodology}

It is a cross-sectional online survey. Total number of participants were: $n=141$. Participants were selected on the basis of inclusion and exclusion criteria. This study includes students currently studying in New Delhi including all genders of age group 18 to 27 years. Informed consent and The Pittsburgh Sleep Quality Index (PSQI) was sent to students via online platform i.e. whatsapp and e-mail. The questionnaire included 7 domains i.e. subjective sleep quality, sleep latency, sleep duration, habitual sleep efficiency, sleep disturbances, use of sleeping medication, and daytime dysfunction.

Participants who were falling under the inclusion criteria and willing to be a part of research were selected. After collecting the responses of students, data was evaluated by the researcher. Any participant not willing to participate was excluded from the study.

Inclusion Criteria were: collegiate students studying in Delhi, participants of both genders, age group of 18 to 27 years. Exclusion criteria were: students who were not willing to participate and who were already diagnosed with any sleeping disorder.

Dependent variable was sleep quality and independent variables were lockdown and COVID-19. Outcome measure was PSQI Scale.

\section{Procedure for PSQI Scale}

PSQI consists of 19 self-rated questions and 5 questions rated by the bed partner or roommate. These 5 questions were for clinical information only. The 19 self-rated questions assess a variety of factors such as sleep quality including an estimate of time duration and latency and frequency of severity of sleep-related problem. These items are grouped into 7 component scores; each weighs 0-3 scale. The ranges of 7 components are from $0-21$. Higher scores indicate worst sleep. The entire index requires 5-10 min for subjects to complete and 5 minutes to score. 


\section{Result}

Data analysis was done via SPSS 2020. Descriptive analysis was done. Analysis of results shows that the mean of age was 23.49 with std. deviation 2.273, gender was 0.29 with std. deviation 0.456 , sleep quality was 1.13 with std. deviation 0.864 , sleep latency was 1.77 with std. deviation 0.974, and sleep efficiency was 0.74 with std. deviation 0.923 , sleep duration was 0.64 with std. deviation 0.973, sleep medication was 1.23 with std. deviation 0.53 , sleep disturbance was 0.09 with std. deviation 0.395 , day dysfunction was 0.87 with std. deviation 0.880 and PSQI was 6.48 with std. deviation 3.200 (Table 1).

Table I.Mean Standard Deviation of Subjects and PSQI Scale

\begin{tabular}{|c|c|c|c|}
\hline & N Statistic & $\begin{array}{c}\text { Mean } \\
\text { Statistic }\end{array}$ & $\begin{array}{c}\text { Mean std. } \\
\text { Deviation }\end{array}$ \\
\hline Age & 141 & 23.49 & 2.273 \\
\hline Gender & 141 & 0.29 & 0.456 \\
\hline Sleep Quality & 141 & 1.13 & 0.864 \\
\hline Sleep Latency & 141 & 1.77 & 0.974 \\
\hline $\begin{array}{c}\text { Sleep } \\
\text { Efficiency }\end{array}$ & 141 & 0.74 & 0.923 \\
\hline Sleep Duration & 141 & 0.64 & 0.973 \\
\hline $\begin{array}{c}\text { Sleep } \\
\text { Medication }\end{array}$ & 141 & 1.23 & 0.530 \\
\hline $\begin{array}{c}\text { Sleep } \\
\text { Dysfunction }\end{array}$ & 141 & 0.09 & 0.395 \\
\hline $\begin{array}{c}\text { Day } \\
\text { Dysfunction }\end{array}$ & 141 & 0.87 & 0.880 \\
\hline \begin{tabular}{c} 
PSQI \\
\hline
\end{tabular} & 141 & 6.48 & 3.200 \\
\hline
\end{tabular}

Maximum numbers of participants were of age 25 years with $23.4 \%$. Male were $29.1 \%$ and female were $70.9 \%$.

Result also shows that $19.1 \%$ were having very good sleep quality whereas $12.1 \%$ were having bad sleep. $60.3 \%$ were having fairly good sleep whereas $8.5 \%$ were having fairly bad sleep. Sleep latency was noted as $8.5 \%$ participants sleep within $<15$ minutes, $35.5 \%$ participants sleep within 16 to 30 minutes, $26.2 \%$ participants take $31-60$ minutes whereas $29.8 \%$ participants take more than 60 minutes top fall asleep. Sleep efficiency was noted as $51.8 \%$ participant was having very good sleep efficiency whereas $7.1 \%$ were having very bad sleep efficiency. $61.7 \%$ participants were having very good sleep duration hours whereas $9.9 \%$ participants were having very bad sleep duration hours. 4.3\% participants don't take sleep medicine in last month whereas $0.7 \%$ has taken medication for sleep for more than thrice in a week. $68.8 \%$ participants have taken sleep medicine once a week and $26.2 \%$ has taken medicine twice a week. $41.1 \%$ participants never have day time dysfunction whereas $5.0 \%$ participants have day time dysfunction for than thrice a week (Figures 1 and 2).

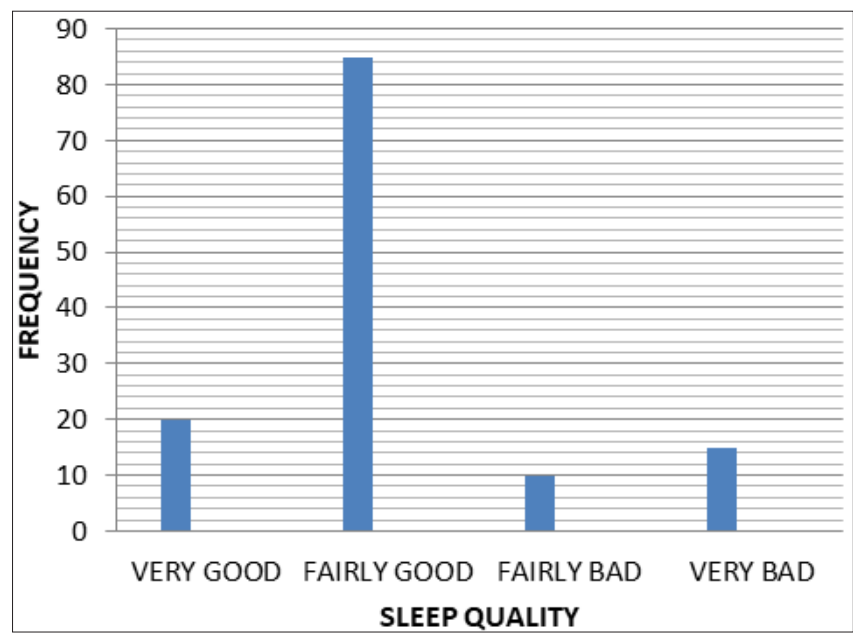

Figure I.Sleep Quality

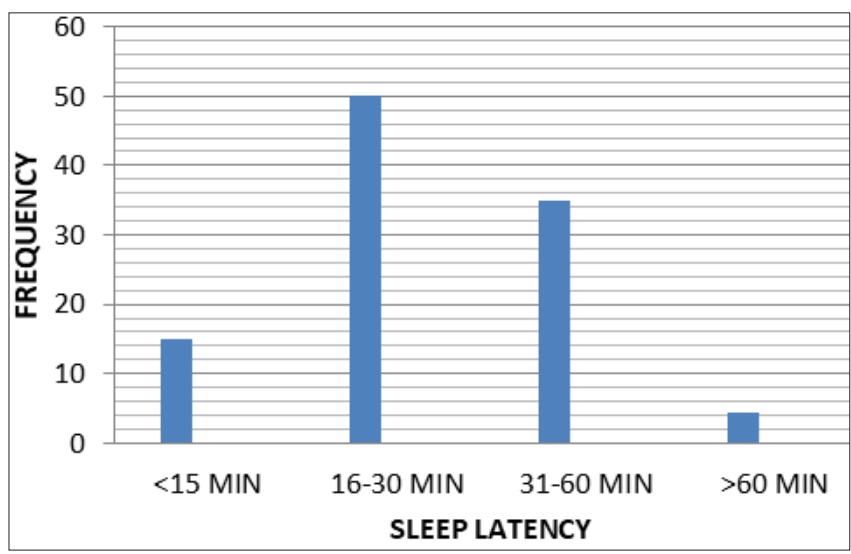

Figure 2.Sleep Latency

\section{Discussion}

In this cross-sectional survey, we have a tendency to study the sleep pattern of collegiate students, sleep-wake rhythms markedly modified, with individual progressing to bed and waking up later, and disbursal longer in bed, but, paradoxically, additionally news a lower sleep quality. These changes are also related to many factors like cut back physical activity, less daylight exposure, increase use of digital technology and psychological distress. ${ }^{12}$ Stress and sleep connected disorders square measures common among population. ${ }^{13}$ A recent review on sleep issue throughout home confinement due to COVID-19 happening by European CBT-I Academy targeted the eye on sleep and sleep disorder. ${ }^{13}$

Cellini et al. assessed changes in sleep pattern, sense of your time, and use of electronical devices in 1310 young adults (workers and university students), and that they found a rise of digital media's use in the evening before bedtime hours. Moreover, they found that individual visited 
the bed and woke later, and spent longer in bed (delayed by -41 minutes), with a lower quality of sleep $(p=1.66)$. Participants with higher levels of depression, anxiety, and stress $(p<0.001)$ showed additional sleep issues. ${ }^{12}$

A study conducted by Li et al. showed that the prevalence of sleep disorder (insomnia) inflated considerably throughout the COVID-19 happening (in some cases new onsets of insomnia), that time in bed (TIB) with more than 66.3 minutes and Total Sleep Time (TST) inflated from 65.6 minutes to 95.6 minutes, in which sleep efficiency considerably potency cut. ${ }^{14}$

When compared to certain studies, ${ }^{12,14}$ our finding shows more inflation in sleep latency $(29.8 \%)$, sleep efficiency (51.8\%) increase sleep duration (61.7\%).

\section{Conclusion}

Lockdown had affected the sleep quality of collegiate students. There is reduction in sleep latency and sleep efficiency and increased bed time. Thus it has been concluded that collegiate students were having a poor quality of sleep amidst the lockdown.

\section{Ethical Issue}

Ethical consent was taken from Ethical commission of Jamia Hamdard University.

\section{Conflict of Interest: None}

\section{References}

1. Pediatr. 2020:87;281-286. [Pubmed] [Google Scholar]

2. Marelli S, Castelnuovo A, Somma A, Castronovo A, Mombelli S, Bottoni D, Leitner, Fossati CA, FeriniStrambi L. Impact of COVID-19 lockdown on sleep quality in university students and administration staff. J Neurol. 2021:268;8-15. [Pubmed] [Google Scholar]

3. Bao Y, Sun Y, Meng S, Shi J, Lu L. 2019-nCoV epidemic: address mental health care to empower society. Lancet. 2020 Feb 22;395(10224):e37-e38. https:// doi.org/10.1016/S0140-6736(20)30309-3. [Pubmed] [Google Scholar]

4. Sarsak HI. Telerehabilitation services: a successful paradigm for occupational therapy clinical services. Int Phys Med Rehab J. 2020:5(2):93-98. DOI: 10.15406/ ipmrj.2020;5:00237. [Pubmed] [Google Scholar]

5. Cao W, Fang Z, Hou G, Han M, Xu X, Dong J. The psychological impact of the COVID-19 epidemic on college students in China. Psychiatry Res. 2020:287(March);112934. [Pubmed] [Google Scholar]

6. Duong V, Luo J, Pham P, Yang T, Wang Y. The ivory tower lost: how college students respond differently than the general public to the COVID-19 Pandemic. ASONAM 2020:126-130. [Google Scholar]

7. Andria Pragholapati. EdARXiv [Preprint]. COVID-19 impact on students, May 6, 2020 [cited 2021 Jan 27]. Available from: https://edarxiv.org/895ed/.

8. Billy $M$. The influence of dynamic organizations and the application of digital innovations to educational institutions in the world during the COVID-2019 [cited 27 Jan 2021]. Available from: https://papers.ssrn. com/sol3/papers.cfm?abstract_id=3588233 [Google Scholar]

9. Chokroverty S. Overview of sleep and sleep disorders. Indian J Med Res. 2010 (Feb);126-140. [Pubmed] [Google Scholar]

10. Buysse DJ, Reynolds $3^{\text {rd }}$ CF, Monk TH, Berman SR, Kupfer DJ. The Pittsburg Sleep Quality Index: A new instrument for psychiatric practice and research. Psychiatry Res. 1989:28;193-213. [Pubmed] [Google Scholar]

11. Smyth C. The Pittsburgh Sleep Quality Index (PSQI). J Gerontol Nurs. 1999;25(12):10. [Google Scholar]

12. Cellini N, Canale N, Mioni G, Costa S. Changes in sleep pattern, sense of time and digital media use during COVID-19 lockdown in Italy. J Sleep Res. 2020:29(4);e13074. https://doi.org/10.1111/jsr.13074. [Pubmed] [Google Scholar]

13. Altena E, Baglioni C, Espie CA, Ellis J, Gavriloff D, Holzinger B, Schlarb A, Frase L, Jernelöv S, Riemann D. Dealing with sleep problems during home confinement due to the COVID-19 outbreak: practical recommendations from a task force of the European CBT-I Academy. J Sleep Res. 2020 Aug;29(4):e13052. https://doi.org/10.1111/jsr.13052. [Pubmed] [Google Scholar]

14. Li Y, Qin Q, Sun Q, Sanford LD, Vgontzas AN, Tang X. (2020) Insomnia and psychological reactions during the COVID-19 outbreak in China. J Clin Sleep Med. https:// doi.org/10.5664/jcsm.8524. [Pubmed] [Google Scholar] 